\title{
EMERGÊNCIA DE SEMENTES CRIOULAS DE MILHO OBTIDAS DE SELEÇÃO FENOTÍPICA ${ }^{1}$
}

\author{
Lucas Alexandre dos Santos Rocha², Olavo José Marques Ferreira², João Pedro Ferreira Santos², Lucas \\ Henrique da Silva Amancio², Michelle Conceição Vasconcelos², Renata Silva-Mann² \\ ${ }^{1} O$ presente trabalho faz parte de projeto PIBIC financiado pela UFS \\ ${ }^{2}$ Universidade Federal de Sergipe - UFS, Campus São Cristóvão, Cidade Univ. Prof. José Aloísio de Campos, São Cristóvão/SE \\ E-mail: Lucas1404_2411@outlook.com; olavojose@hotmail.com; joao20pedro@outlook.com; lucashenrique.ama@gmail.com; \\ michelle_florestal@yahoo.com.br; renatamann@gmail.com
}

\begin{abstract}
RESUMO: O milho crioulo é de grande importância para as comunidades tradicionais, pois possui um custo menor de implantação e manutenção, sendo uma semente adaptada e produzida por pequenos produtores 0 que the agrega relevante importância social. 0 objetivo deste trabalho foi caracterizar duas variedades crioulas de milho "pé de boi" e "paulistão" utilizando como base as características fisiológicas das sementes: índice de velocidade de emergência (IVE); tempo médio de germinação (TM); massa seca de plântulas (MSP); comprimento de plântulas (CP); porcentagem de plântulas normais (\% PN) e peso de cem sementes (P100). Com base nos resultados obtidos foi possível identificar uma diferença entre o peso de 100 sementes quando a população do "paulistão" apresentou maior peso, porém menor IVE. O tempo médio de emergência não diferiu entre as matrizes. A variedade "pé de boi" apresenta maior vigor. Para o desenvolvimento inicial, as matrizes 13 e 14 de "pé de boi" foram superiores.
\end{abstract}

Palavras-chave: Zea mays L., variedade tradicional, seleção massal, melhoramento

\section{EMERGENCE OF CORN SEEDLINGS OBTAINED FROM PHENOTYPIC SELECTION}

\begin{abstract}
Creole maize is of great importance for traditional communities, since it has a lower cost of implantation and maintenance, being a seed adapted and produced by small producers which adds significant social importance. The objective of this work was to characterize two native varieties of "pé de boi" and "paulistão" using the physiological characteristics of the seeds: emergency speed index (IVE); mean germination time (TM); dry mass of seedlings (MSP); length of seedlings (CP); percentage of normal seedlings (\% PN) and weight of one hundred seeds (P100). Based on the results obtained, it was possible to identify a difference between the weight of 100 seeds when the population of "paulistão" presented higher weight, but lower IVE. The mean time of emergence did not differ between matrices. The variety "pé de boi" presents greater vigor. For initial development, the "pé de boi" matrices 13 and 14 were superior.
\end{abstract}

Keywords: Zea mays L., traditional variety, mass selection, breeding

\section{INTRODUÇÃO}

$O$ consumo de milho (Zea mays L.) na região Nordeste do Brasil é realizado de diferentes formas na culinária tradicional. Para

suprir a demanda por alimento cada vez mais os agricultores têm investido no cultivo do cereal, sendo a semente o principal insumo. Entretanto, pequenos agricultores não dispõem de recursos financeiros para aquisição do insumo e todo o pacote tecnológico associado, fazendo uso de variedades crioulas pelo seu menor custo.
Cultivado em menor escala, as sementes de milho de variedades crioulas são produzidas pelo próprio agricultor ou comunidade rural in situ ou on farm, a partir de sucessivas seleções fenotípicas, sendo chamadas também de cultivar local ou tradicional.

0 uso destas sementes, além de garantir segurança alimentar, representa uma estratégia de conservação, fundamental para a manutenção da diversidade biológica e da variabilidade genética; fatores importantes para o melhoramento da espécie. (PATERNIANI et al., 2000) 
A legislação vigente não estabelece 0 controle de qualidade para o uso de sementes produzidas pelo próprio agricultor. Podendo 0 agricultor utilizar variedades cruzadas com baixo vigor. Neste sentido, o objetivo deste trabalho foi avaliar a emergência e vigor de sementes crioulas obtidas de seleção fenotípica.

\section{MATERIAL E MÉTODOS}

As variedades Paulistão e Pé de boi são cultivadas em Sergipe e as sementes foram obtidas na safra 2018 em área experimental no Campus Rural da Universidade Federal de Sergipe (UFS) em São Cristóvão. O clima é do tipo As', segundo Köppen (1948) caracterizado por verão seco e pluviometria de $1200 \mathrm{~mm}$ anuais com chuvas concentradas nos meses de abril e setembro. Os dados pluviométricos do período foram obtidos na estação meteorológicas do local e apresentados na Figura 1.

Figura 1. Precipitação e temperatura do Campus Rural da Universidade Federal de Sergipe, durante o período de cultivo das variedades de milho crioulo.

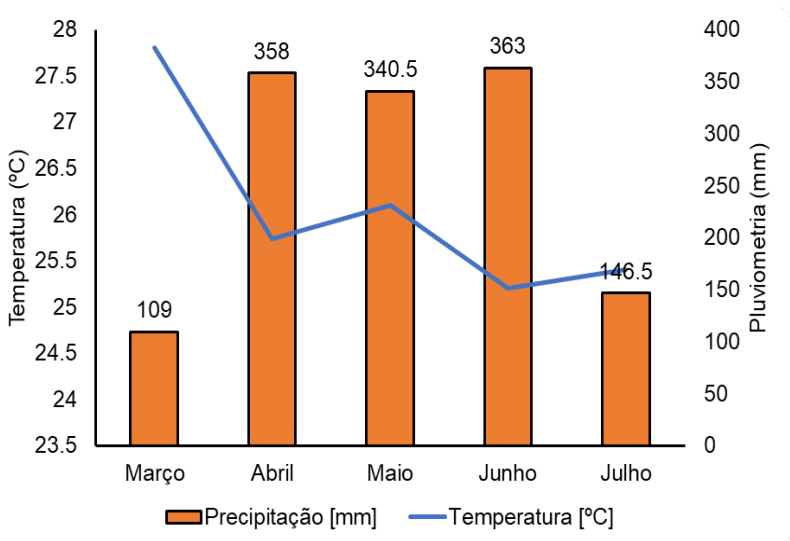

As variedades de milho foram cultivadas para a seleção fenotípica de plantas produtivas, com intensidade de seleção de $10 \%$. Originando doze matrizes de paulistão e quinze de pé de boi organizadas em ordem decrescente neste teste.

As sementes das matrizes selecionadas, foram colhidas no ponto de maturidade fisiológica e secas em estufa termoelétrica a uma temperatura de $40^{\circ} \mathrm{C}$ até que atingissem $13 \%$ de umidade (b.u.). Para a separação das matrizes em diferentes classes de vigor, foram realizadas avaliações fisiológicas. As avaliações foram: peso de cem sementes (P100); porcentagem de plântulas normais (\%PN); índice de velocidade de emergência (IVE); tempo médio de emergência (TM); massa seca (MSP) e altura de plântulas (AP) e comprimento de radículas (CR).

Para a determinação da P100 foi utilizada uma balança analítica conforme metodologia da Regra para Análise de Sementes (Brasil, 2009). 0 teste de emergência foi realizado em casa de vegetação, em bandejas de polietileno, contendo areia lavada (substrato) e mantendo a umidade em $60 \%$ da capacidade máxima de retenção de água. Cada parcela (27 matrizes) era composta por quatro repetições de 25 sementes.

Foram realizadas contagens diárias da emergência para a obtenção do IVE, TM, \%PN. Para o cálculo do IVE utilizou-se a fórmula sugerida por Maguire (1962). A emergência de plântulas foi contabilizada aos quatro e sete dias após a semeadura.

Para altura de plântulas e comprimento de radículas, foram selecionadas, aleatoriamente, 15 plântulas normais de cada matriz de milho aos nove dias após a semeadura. A medição foi realizada com uma régua graduada em centímetros.

A massa seca de plântulas foi determinada após a lavagem e retirada do tecido de reserva e a secagem por 24 horas em estufa termoelétrica a $80^{\circ} \mathrm{C}$. A pesagem do material foi feita em balança de precisão, 0,001 g, com massa expressa em mg plântulas-1.

Os dados obtidos foram submetidos à análise de variância e as médias agrupadas pelo teste de ScottKnott, a $5 \%$ de probabilidade. A análise foi realizada com o auxílio do programa estatístico SISVAR® (FERREIRA, 2011).

\section{RESULTADOS E DISCUSSÃO}

As sementes de paulistão apresentaram média da massa de cem sementes superior às sementes do Pé de Boi, importando um maior volume de sementes (Figura 2). 
Figura 2. Peso de cem sementes das variedades de milho crioulo utilizadas no teste. Universidade Federal de Sergipe - UFS, São cristóvão, SE, 2018.

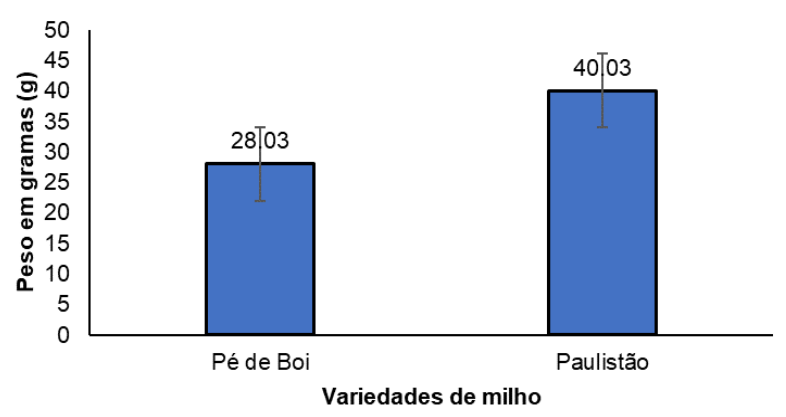

Para o índice de velocidade de emergência, verificou-se que na variedade pé de boi, as sementes oriundas das plantas de menor desempenho no campo, tiveram maiores valores (Tabela 1).
As matrizes pé de boi 13 e 14 apresentaram maior vigor que as demais. Para milho, germinação em 48 horas é considerado rápido (FAVERO et al., 2001). Entretanto, tempos superiores a 120 horas caracterizam atraso na germinação (SARTOR et al., 2015), como nas sementes de paulistão que tiveram menor valor de IVE.

Verificou-se que o tempo médio de emergência de plântulas não diferiu estatisticamente entre as variedades e variou entre 5,99 e 6,26 dias.

Aemergência de plântulas normais de pé de boi demonstrou que as sete melhores plantas no campo, produziram sementes com baixo desempenho, exceto a pé de boi 4. As plântulas de Paulistão apresentaram emergência superior a pé de boi, exceto para Paulistão 2, 6 e 11.

Tabela 1. Índice de Velocidade de Emergência (IVE), tempo médio de emergência (TM) e plântulas normais (\%PN) de duas variedades de sementes de crioulo, pé de boi e paulistão.

\begin{tabular}{|c|c|c|c|}
\hline Matrizes & IVE & $\mathrm{TM}$ & PN (\%) \\
\hline Pé de Boi 1 & $11.79 b$ & $6.15 a$ & $79.00 \mathrm{~b}$ \\
\hline Pé de Boi 2 & $8.54 \mathrm{c}$ & $6.23 \mathrm{a}$ & $82.00 \mathrm{~b}$ \\
\hline Pé de Boi 3 & $6.86 \mathrm{c}$ & $6.32 \mathrm{a}$ & $79.00 \mathrm{~b}$ \\
\hline Pé de Boi 4 & $11.61 b$ & $6.08 \mathrm{a}$ & $98.00 \mathrm{a}$ \\
\hline Pé de Boi 5 & $8.31 \mathrm{c}$ & $6.12 \mathrm{a}$ & $83.00 \mathrm{~b}$ \\
\hline Pé de Boi 6 & $9.23 c$ & $6.22 \mathrm{a}$ & $87.00 \mathrm{~b}$ \\
\hline Pé de Boi 7 & $9.68 \mathrm{c}$ & $6.15 a$ & $88.00 \mathrm{~b}$ \\
\hline Pé de Boi 8 & $12.23 b$ & $6.05 a$ & $97.00 \mathrm{a}$ \\
\hline Pé de Boi 9 & $12.65 b$ & $6.03 a$ & $99.00 \mathrm{a}$ \\
\hline Pé de Boi 10 & $15.71 \mathrm{a}$ & $5.99 a$ & $93.00 \mathrm{a}$ \\
\hline Pé de Boi 11 & $11.07 \mathrm{c}$ & $6.07 \mathrm{a}$ & $90.00 \mathrm{a}$ \\
\hline Pé de Boi 12 & $12.01 \mathrm{~b}$ & $6.12 \mathrm{a}$ & $100.00 \mathrm{a}$ \\
\hline Pé de Boi 13 & $13.85 b$ & $6.00 \mathrm{a}$ & 99.00 a \\
\hline Pé de Boi 14 & 15.41 a & $6.07 \mathrm{a}$ & $100.00 \mathrm{a}$ \\
\hline Pé de Boi 15 & $17.06 \mathrm{a}$ & $6.04 \mathrm{a}$ & $99.00 \mathrm{a}$ \\
\hline Paulistão 1 & $8.95 \mathrm{c}$ & $6.26 \mathrm{a}$ & $97.00 \mathrm{a}$ \\
\hline Paulistão 2 & $7.96 \mathrm{c}$ & $6.21 \mathrm{a}$ & $81.00 \mathrm{~b}$ \\
\hline Paulistão 3 & $9.90 \mathrm{~b}$ & $6.20 \mathrm{a}$ & 98.00 a \\
\hline Paulistão 4 & $8.78 \mathrm{c}$ & $6.27 \mathrm{a}$ & $97.00 \mathrm{a}$ \\
\hline Paulistão 5 & $9.60 \mathrm{c}$ & $6.21 \mathrm{a}$ & $95.00 \mathrm{a}$ \\
\hline Paulistão 6 & $8.42 \mathrm{c}$ & $6.23 a$ & $89.00 \mathrm{~b}$ \\
\hline Paulistão 7 & $9.38 \mathrm{c}$ & $6.17 \mathrm{a}$ & $100.00 \mathrm{a}$ \\
\hline Paulistão 8 & $9.07 \mathrm{c}$ & $6.23 a$ & $94.00 \mathrm{a}$ \\
\hline Paulistão 9 & $9.02 \mathrm{C}$ & $6.24 \mathrm{a}$ & $92.00 \mathrm{a}$ \\
\hline Paulistão 10 & $10.37 \mathrm{c}$ & $6.16 \mathrm{a}$ & $99.00 \mathrm{a}$ \\
\hline Paulistão 11 & $7.29 \mathrm{c}$ & $6.19 a$ & $73.00 \mathrm{~b}$ \\
\hline Paulistão 12 & $9.77 \mathrm{c}$ & $6.21 \mathrm{a}$ & $97.00 \mathrm{a}$ \\
\hline CV\% & 23.22 & 3.74 & 13.94 \\
\hline
\end{tabular}

*Médias seguidas pela mesma letra, na coluna, não diferem entre si pelo teste de Scott-knott ao nível de 5\% de probabilidade. 
Observou-se que as maiores altura de mais vigorosas (NAKAGAWA, 1999), as plântulas de plântulas e comprimento de radícula são na maioria da pé de boi são mais vigorosas que as de paulistão. A variedade pé de boi. Tendo em vista que as amostras matriz paulistão 6 foi a que obteve maiores valores de que apresentam os maiores valores médios são as altura e comprimento (Tabela 2).

Tabela 2. Altura de Plântulas (AP- cm), comprimento de radícula (CR- cm) e massa seca de plântulas (MSP- $\mathrm{mg}$ ) de duas variedades de sementes de milho crioulo, pé de boi e paulistão.

\begin{tabular}{llll} 
Matrizes & \multicolumn{1}{c}{$\mathrm{CP}(\mathrm{cm})$} & $\mathrm{CR}(\mathrm{cm})$ & MSP $(\mathrm{mg})$ \\
Pé de Boi 1 & $14.48 \mathrm{a}$ & $9.57 \mathrm{c}$ & $0.99 \mathrm{~b}$ \\
Pé de Boi 2 & $7.63 \mathrm{~b}$ & $3.85 \mathrm{c}$ & $0.68 \mathrm{~b}$ \\
Pé de Boi 3 & $9.53 \mathrm{~b}$ & $6.63 \mathrm{c}$ & $0.90 \mathrm{~b}$ \\
Pé de Boi 4 & $15.23 \mathrm{a}$ & $8.36 \mathrm{c}$ & $1.48 \mathrm{a}$ \\
Pé de Boi 5 & $9.77 \mathrm{~b}$ & $4.81 \mathrm{c}$ & $0.77 \mathrm{~b}$ \\
Pé de Boi 6 & $7.66 \mathrm{~b}$ & $3.03 \mathrm{c}$ & $0.87 \mathrm{~b}$ \\
Pé de Boi 7 & $12.96 \mathrm{a}$ & $8.41 \mathrm{c}$ & $1.24 \mathrm{a}$ \\
Pé de Boi 8 & $11.55 \mathrm{a}$ & $7.55 \mathrm{c}$ & $0.88 \mathrm{~b}$ \\
Pé de Boi 9 & $14.31 \mathrm{a}$ & $8.16 \mathrm{c}$ & $1.51 \mathrm{a}$ \\
Pé de Boi 10 & $14.21 \mathrm{a}$ & $9.22 \mathrm{c}$ & $1.54 \mathrm{a}$ \\
Pé de Boi 11 & $9.01 \mathrm{~b}$ & $4.31 \mathrm{c}$ & $0.74 \mathrm{~b}$ \\
Pé de Boi 12 & $12.38 \mathrm{a}$ & $7.31 \mathrm{c}$ & $1.38 \mathrm{a}$ \\
Pé de Boi 13 & $16.35 \mathrm{a}$ & $19.12 \mathrm{a}$ & $1.60 \mathrm{a}$ \\
Pé de Boi 14 & $14.45 \mathrm{a}$ & $22.75 \mathrm{a}$ & $1.53 \mathrm{a}$ \\
Pé de Boi 15 & $13.82 \mathrm{a}$ & $6.60 \mathrm{c}$ & $1.09 \mathrm{~b}$ \\
Paulistão 1 & $9.51 \mathrm{~b}$ & $12.30 \mathrm{~b}$ & $1.36 \mathrm{a}$ \\
Paulistão 2 & $6.37 \mathrm{~b}$ & $11.03 \mathrm{~b}$ & $1.67 \mathrm{a}$ \\
Paulistão 3 & $7.80 \mathrm{~b}$ & $12.33 \mathrm{~b}$ & $1.62 \mathrm{a}$ \\
Paulistão 4 & $8.02 \mathrm{~b}$ & $13.07 \mathrm{~b}$ & $1.50 \mathrm{a}$ \\
Paulistão 5 & $9.06 \mathrm{~b}$ & $13.25 \mathrm{~b}$ & $1.93 \mathrm{a}$ \\
Paulistão 6 & $12.71 \mathrm{a}$ & $10.94 \mathrm{~b}$ & $1.29 \mathrm{a}$ \\
Paulistão 7 & $10.06 \mathrm{~b}$ & $13.15 \mathrm{~b}$ & $1.48 \mathrm{a}$ \\
Paulistão 8 & $7.53 \mathrm{~b}$ & $11.08 \mathrm{~b}$ & $1.25 \mathrm{a}$ \\
Paulistão 9 & $7.41 \mathrm{~b}$ & $12.31 \mathrm{~b}$ & $1.37 \mathrm{a}$ \\
Paulistão 10 & $7.90 \mathrm{~b}$ & $10.31 \mathrm{~b}$ & $1.32 \mathrm{a}$ \\
Paulistão 11 & $11.54 \mathrm{~b}$ & $1.53 \mathrm{a}$ \\
Paulistão 12 & $13.31 \mathrm{~b}$ & $13.82 \mathrm{~b}$ & $1.42 \mathrm{a}$ \\
CV\% & $10.92 \mathrm{~b}$ & 41.07 & 23.59
\end{tabular}

*Médias seguidas pela mesma letra, na coluna, não diferem entre si pelo teste de Scott-knott ao nível de 5\% de probabilidade.

0 comprimento radicular foi maior nas plântulas de pé de boi 13 e 14 apresentando um maior desenvolvimento radicular o que implica em um crescimento e absorção de nutrientes melhor (BARBER, 1988).

Foi verificado que as matrizes de pé de boi 4,7 , 9, 13 e 14 obtiveram maior massa seca de plântulas. As sementes vigorosas proporcionam maior transferência de massa seca de seus tecidos de reserva para o eixo embrionário, na fase de germinação originando plântulas com maior peso em função do maior acúmulo de matéria (NAKAGAWA, 1999). A massa seca das plântulas das matrizes de paulistão não diferiu entre si.

\section{CONCLUSÕES}

A variedade pé de boi por obter melhores resultados para a maioria das características fisiológicas avaliadas (AP, CR, MSP e IVE) é a indicada para possíveis estudos de melhoramento. 


\section{REFERÊNCIAS}

BARBER, S. A.; MACKEY, A. D.; KUCHENBUCH, R. O.; BARRACLOUGH, S. Effect of soil temperature and water on maize root growth. Plant and Soil, 1988,111, 267-269.

BRASIL, Ministério da Agricultura e reforma Agrária. Regras para análises de sementes. Brasilia: SNDA/ DNDV/CLAV, 2009. 398p.

EDMOND, J. B.; DRAPALA, W. J. The effects of temperature, sand and soil, and acetone on germination of okra seed. Proceedings of the American Society for Horticultural Science, 1958, 71, 428-443.

FERREIRA, D. F. Sisvar: a computer statistical analysis system. Ciência e Agrotecnologia, 2011, 35, 1039-1042.

KOEPPEN, W. Climatologia: com un estudio de los climas de la tierra. Fondo de Cultura Econômica. México. 1948. 479p.
MAGUIRE, J. D. Speed of germination aid in selection and evaluation for seedling emergence and vigor. Crop Science, 1962, 2, 176-77, 1962.

NAKAGAWA, J. Testes de vigor baseados na avaliação de plântulas. In: KRZYZANOWSKI, F.C.; VIEIRA, R.D.; FRANÇA-NETO, J.B. (Ed.). Vigor de sementes: conceitos e testes. Londrina: ABRATES, 1999. p.2-21.

PATERNIANI, E.; NASS, L. L.; SANTOS, M.X. O valor dos recursos genéticos de milho para o Brasil: uma abordagem histórica da utilização do germoplasma. In: UDRY, C. W.; DUARTE, W. (Org.) Uma história brasileira do milho: 0 valor dos recursos genéticos. Brasília: Paralelo 15, p.11-41, 2000.

SARTOR, L. R.; LOPES, L.; MARTIN, T. N.; ORTIZ, $S$. Alelopatia de acículas de pínus na germinação e desenvolvimento de plântulas de milho, picão preto e alface. Bioscience Journal, 2015, 31, 470-480. 